\title{
Synergistic Antifungal Effect of Fluconazole Combined with Zno Nanoparticles against Candida albicans Strains from Vaginal Candidiasis
}

\author{
Azadeh Abedzadeh Hajar (PhD \\ Candidate) \\ Department of Microbiology, Qom \\ Branch, Islamic Azad University, \\ Qom, Iran \\ Mohammad Dakhili (PhD) \\ Department of Lab Science, \\ Faculty of Medicine, Qom Branch, \\ Islamic Azad University, Qom, \\ Iran \\ Mojgan Saghazadeh (PhD) \\ Department of Microbiology, Qom \\ Branch, Islamic Azad University, \\ Qom, Iran \\ Soheil Aghaei (PhD) \\ Department of Microbiology, Qom \\ Branch, Islamic Azad University, \\ Qom, Iran., Iran \\ Razieh Nazari (PhD) \\ Department of Microbiology, Qom \\ Branch, Islamic Azad University, \\ Qom, Iran \\ Corresponding author: \\ Mohammad Dakhili \\ E-mail: dr_dakhili@yahoo.com \\ Tel: 09124532454 \\ Address: Department of Lab \\ Science, Faculty of Medicine, \\ Qom Branch, Islamic Azad \\ University, Qom, Iran \\ Received: 2019/05/27 \\ Revised: 2019/07/29 \\ Accepted: 2019/07/31 \\ (c) (i) (9)
}

This work is licensed under a Creative Commons Attribution 4.0 License.

\begin{abstract}
Background and Objective: The prevalence of the infections caused by Candida species has led to a significant increase in their resistance to antifungal compounds. The aim of this study was to i) investigate drug resistance ii) evaluate the incidence of Candida albicans drug resistance pattern in the vaginal samples of women referring to health centers of Qom province, and iii) examine the effect of $\mathrm{Zn}$ nanoparticles combined with fluconazole against $\mathrm{C}$. albicans isolates.

Methods: This experimental, descriptive study was performed on 120 patients of candidiasis. In order to identify Candida albicans, direct experiments, differential culture, Germ tube test and sugar assimilation test (API20C kit) were conducted. The effect of different antifungal drugs and zinc nanoparticles and the synergistic effect of fluconazole with zinc nanoparticles were investigated by disk diffusion method. Minimum Inhibitory Concentration (MICs) of all cases was further specified.
\end{abstract}

Results: Of the 120 samples, $50(41.6 \%)$ were identified as Candida albicans. These strains were resistant to certain antifungal drugs while others were semi-sensitive and sensitive. The lowest and the highest mean diameter of inhibition zone in all Candida albicans isolates belonged to ketoconazole $(15.64 \mathrm{~mm})$ and fluconazole nano-ZnO $(26.76 \mathrm{~mm})$, respectively. The lowest and the highest MICs were observed in fluconazole- nano- $\mathrm{ZnO}$ and nano- $\mathrm{ZnO}$, respectively.

Conclusion: The synergistic effect of $\mathrm{Zn}$ nanoparticles with fluconazole can be conducive to the treatment of vaginal candidiasis.

Keywords: Candida albicans, Nanoparticles, Antifungal Agents, Candidiasis, Genital vulvovaginal

This paper should be cited as: Abedzadeh Hajar A, Dakhili M, Saghazadeh M, Aghaei S, Nazari R. [Synergistic Antifungal Effect of Fluconazole Combined with ZnO Nanoparticles against Candida albicans Strains from Vaginal Candidiasis]. mljgoums. 2020; 14(3): 26-32. 


\section{INTRODUCTION}

As one of the most prevalent fungal infections in humans, candidiasis is caused by various species of Candida, particularly Candida albicans (1). Candida albicans is part of the natural microflora or microorganisms typically living in or on our bodies. This pathogenic yeast can be found in the gastrointestinal (GI) tract, mouth, and vagina (2). Candidiasis has been reported in all cities of Iran. In fact, the host responses to the disease change from minor itching and inflammation to chronic and acute granulomatous (3). This infection is the fourth most prevalent cause of nosocomial infections $(3,4)$. Nystatin, ketoconazole, and fluconazole are employed in the treatment of candidate vaginitis. As a problematic chronic infection in women, Candida albicans is resistant to vaginal nystatin, hence its long and costly treatment process (5). Over the recent years, the discovery and identification of putative anti-fungal agents have altered the perceptions of many mycologists regarding certain drugs. Nanotechnology is searching for ways to overcome pathogens and control and cure diseases. In medicine, drug targeting with nanoparticles has received a great deal of attention, especially in the diagnosis and treatment of various diseases. While antibiotics only prevent a very small number of factors contributing to diseases, a nanoparticle is capable of eliminating a variety of bacteria (6).

For centuries, metals have been utilized as bactericidal and bacteriostatic agents. Among these metals, mention can be made of zinc, gold, copper, and silver, each with a different property and range of performance. Nowadays, nanoparticles are commercialized as antimicrobial and anti-fungal agents. Since the recognition of the resistance of microorganisms to antibiotics, the health care industry has greatly identified the antimicrobial properties of metals, particularly zinc. Protein and DNA damage and cell wall destruction are the main mechanisms associated with the effects of nanoparticles on microorganisms. Nanoparticles containing zinc eliminate bacteria in low concentrations and do not exert any toxic effects on human cells (7).Zinc has great anti-microbial properties in nano-scales compared to larger metal scales. Accordingly, the present study aimed to evaluate the antibiotic pattern and synergistic effect of nanoparticles containing zinc with fluconazole on Candida albicans isolated from vulvovaginal patients in Qom province.

\section{MATERIALS AND METHODS}

This experimental, descriptive study was carried out on 120 patients suspected of Candida infection with vulvovaginitis clinical symptoms as well as asymptomatic referrals to health centers in Qom province. A gynecologist performed the sampling through the use of sterilized swabs; afterwards, the swabs were soaked in tubes containing $1 \mathrm{ml}$ of sterile physiology serum and immediately transmitted to the laboratory.

Ethical approval was obtained from the institutional review board of Qom Islamic Azad University. Written informed consent was obtained from all patients included in the study. To isolate the yeasts, all specimens were cultured on Sabouraud Dextrose Agar (SDA) medium containing chloramphenicol. The plates were incubated at $30^{\circ} \mathrm{C}$ for 24-48 hours, and direct gram staining was performed for the evaluation of the yeasts (8). The yeasts were cultured on Chromogenic Candida Agar (CCA), and after growth, they were cultured on Corn Meal agar (CMA) medium containing Tween 80; the presence of chlamydoconidia and pseudo hyphae was then investigated (9). Green colonies on the CCA medium (causing the chlamydoconidia) were considered as Candida albicans in a microscopic examination of the CMA medium containing Tween 80 (10). Sugar assimilation test, including glucose, maltose, sucrose, trehalose, cellobiose, and raffinose was done using the API20C kit (Biomerieux SA, France) (11). An antibiogram test was performed using standard disc diffusion method for 50 Candida albicans positive samples (12). To do the test, massive culture was carried out by sterilized swabs impregnated with fungal suspension in accordance with an opacity of half the McFarland tube on a Mueller-Hinton agar medium. After plate drying at $37^{\circ} \mathrm{C}$ for 15 minutes, antifungal disks containing fluconazole, ketoconazole, nystatin, Iitraconazole, and amphotericin B were applied. The plates were incubated for 24 hours at $30^{\circ} \mathrm{C}$, and the diameter of the inhibition zone was interpreted according to the manufacturer's table. 
Candida albicans ATCC 10231 was employed as the positive control $(13,14)$. The sensitivity and resistance of the isolated Candida samples to antifungal drugs were reported according to the CLSI standard (diffusion disk) (Table 1).

The zinc oxide nanoparticles (nano- $\mathrm{ZnO}$ ) were synthesized by a wet chemical method using zinc chloride and sodium hydroxide. In this method, $10 \mathrm{cc}$ of zinc chloride solution (0.4 $\mathrm{mol} / \mathrm{L}$ ) was poured into a container, and the aqueous solution of sodium hydroxide $(0.8$ $\mathrm{mol} / \mathrm{L}$ ) was added dropwise to the zinc chloride in the container and simultaneously stirred under electromagnetic agitation for 10 min in order that the zinc chloride was properly dissolved. After 2 hours, the solution was centrifuged and the obtained precipitate was washed five times with distilled water. Next, the precipitates were dried at $400{ }^{\circ} \mathrm{C}$ to obtain zinc oxide nanoparticles (15).

To determine the inhibition zone diameter $(\mathrm{mm})$ of the tested fungi against the nano- $\mathrm{ZnO}$, the disk diffusion method was employed based on CLSI standard. From a fungal suspension an equivalent $0.5 \mathrm{McF}$ arland prepared and from 24-hour culture; the fungi were then examined by sterile swabs on Sabouraud dextrose agar; after that, $20 \mu \mathrm{l}$ of zinc nanoparticle stock was added in 200,500, and $1000 \mu \mathrm{g}$ concentrations to $6 \mathrm{~mm}$ blank sterilized disks; it was then put in an incubator via sterile pins in the vicinity of the flame placed on the medium (16).

Broth macrodilution method was used to determine the MIC. 100- $\mu$ l of Sabouraud dextrose broth medium was added to each plate well, and $100 \mu \mathrm{l}$ of fluconazole $(1024 \mu \mathrm{g}$ / $\mathrm{ml})$ and nano-ZnO $(1000 \mu \mathrm{g} / \mathrm{ml})$ were both separately and simultaneously added to the first well. Afterwards, $100 \mu \mathrm{l}$ of the first well was picked up and added to the next well, a procedure done successively. Then, $10 \mu$ of fungal suspension was added to each well. Two wells were considered as positive and negative controls. The positive control was a medium with $10 \mu \mathrm{l}$ of fungal suspension, and the negative control included a medium with an antifungal that had no growth. Following incubation for 24 hours, the fungal growth was specified by a spectrophotometer at $600 \mathrm{~nm}$; the lowest concentration of antifungal compound inhibiting the growth of the tested fungi, was reported as MIC (17). All statistical analyses were performed using SPSS v.22.

\section{RESULTS}

Out of the 120 patients suspected of Candida infection with or without symptoms of vulvovaginal, 50 Candida albicans species $(41.6 \%)$ were isolated, and 70 cases $(58.4 \%)$ were negative. The results of carbohydrate utilization tests using the API20C kit are shown in Table 2.

In order to investigate the crystalline structure of nano- $\mathrm{ZnO}$ and detect the zinc oxide nanoparticles, XRD (X-ray diffraction) method and a Scanning Electron microscope (SEM) were employed, respectively (Fig. 1 and 2). Based on Fig. 2, the average particle size of the synthesized nanoparticles was $35 \mathrm{~nm}$, and the shape and porosity of the nanoparticles (\%) were almost uniform (spherical and white) and $>99$, respectively.

Resistance or sensitivity of the isolated Candida albicans strains to certain antifungal and nano-ZnO compounds was investigated. The results showed that these strains were resistant to the antifungal compounds, and others were semi-sensitive and sensitive. The highest and the lowest sensitivity of the isolated Candida albicans to the antifungal drugs belonged to amphotericin B (100\%) and ketoconazole (60\%), respectively (Fig. 3).

Table 1. CLSI standard (disc diffusion) to antifungal drugs

\begin{tabular}{lccc}
\hline antifungal drugs & resistance & intermediate & Sensitive \\
\hline Fluconazole 25 & $\leq 14 \mathrm{~mm}$ & $15-18 \mathrm{~mm}$ & $\geq 19 \mathrm{~mm}$ \\
\hline Ketoconazole 10 & $\leq 10 \mathrm{~mm}$ & $11-14 \mathrm{~mm}$ & $\geq 15 \mathrm{~mm}$ \\
\hline Nystatin 10 & $\leq 13 \mathrm{~mm}$ & $14-20 \mathrm{~mm}$ & $\geq 21 \mathrm{~mm}$ \\
\hline Itraconazole 10 & $\leq 13 \mathrm{~mm}$ & $14-22 \mathrm{~mm}$ & $\geq 23 \mathrm{~mm}$ \\
\hline Amphotericin B10 & $\leq 10 \mathrm{~mm}$ & $11-14 \mathrm{~mm}$ & $\geq 15 \mathrm{~mm}$ \\
\hline
\end{tabular}


Table 2. Identification of Candida albicans species

\begin{tabular}{|c|c|c|c|c|c|c|c|c|c|c|c|}
\hline Candida & Raffinose & Cellobiose & Trehalose & Lactose & Sucrose & Maltose & Glucose & $\begin{array}{c}\text { Colony } \\
\text { color on } \\
\text { chromium } \\
\text { agar } \\
\text { medium }\end{array}$ & $\begin{array}{c}\text { Chlamydoconidia } \\
\text { production }\end{array}$ & $\begin{array}{c}\text { Production } \\
\text { of tube } \\
\text { mass }\end{array}$ & $\begin{array}{c}\text { Isolated } \\
\text { candidate } \\
\text { number }\end{array}$ \\
\hline $\begin{array}{l}\text { Candida } \\
\text { albicans }\end{array}$ & - & - & + & - & + & + & + & green & + & + & $1-50$ \\
\hline
\end{tabular}

Figure1. XRD patterns of zinc oxide nanoparticles. $\theta$ shows the X-ray angle reaction with the zinc oxide crystalline plates

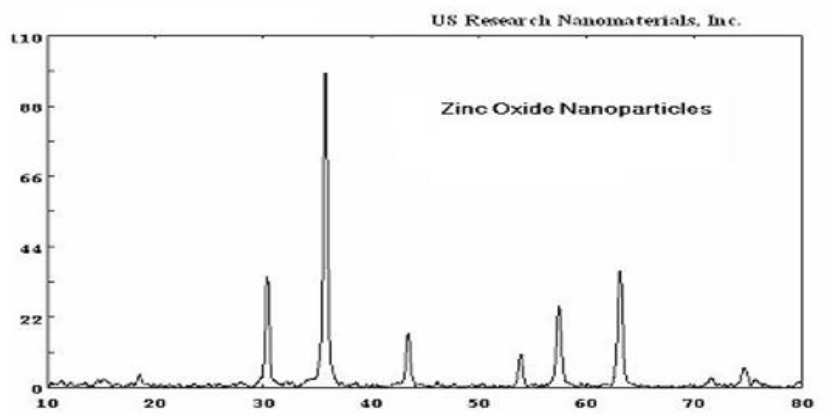

Figure 2. Resistance and sensitivity of Candida albicans to antifungal drugs

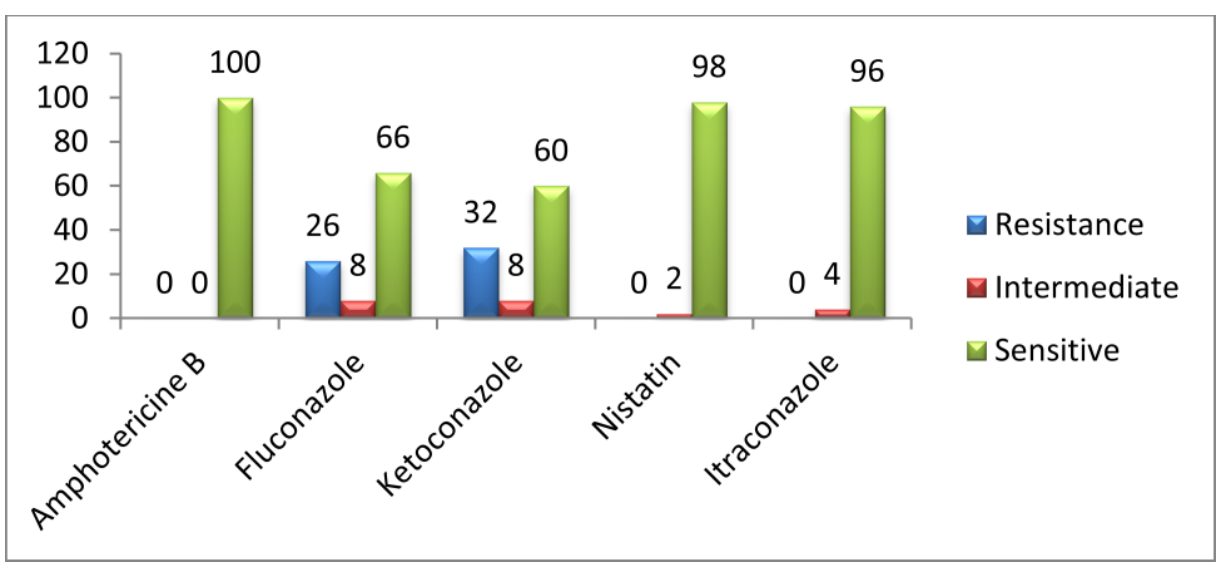

Figure 3: Inhibition zone of Candida albicans strains isolated from the antifungal compounds

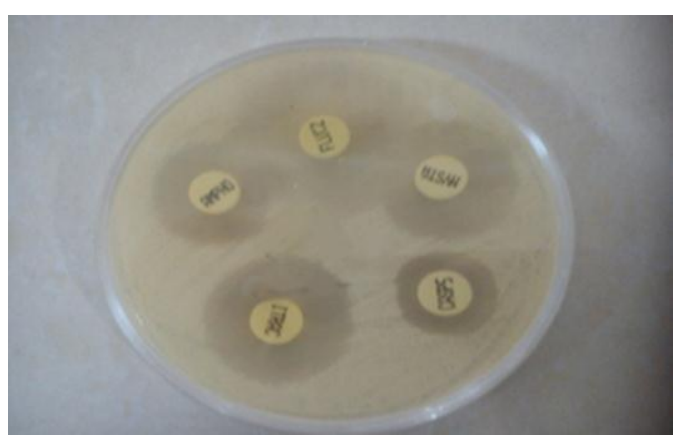


Figure 4: Disk image of disk diffusion with the nanoparticle and fluconazole A: Nano-ZnO, B: Fluconazole, C: Nano-ZnO + Fluconazole

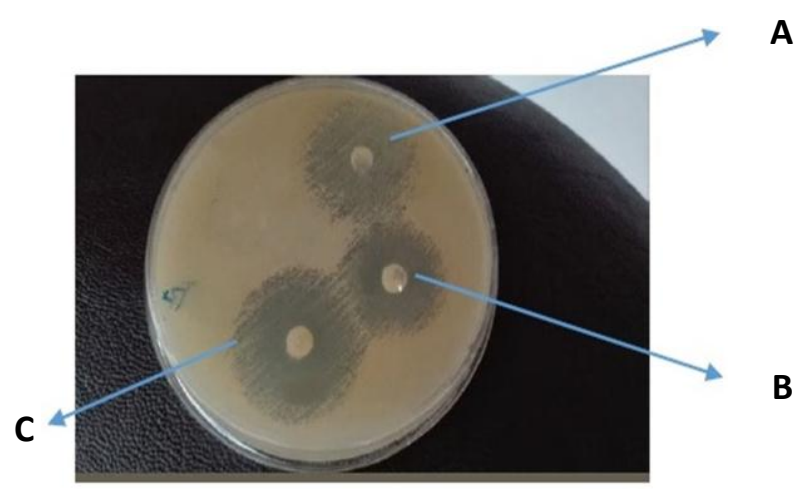

Table 3. Resistance pattern of antifungal compounds used in Candida albicans strains isolated from patients

\section{MIC results}

The lowest and highest MICs belonged to fluconazole nano- $\mathrm{ZnO}$ and nano- $\mathrm{ZnO}$, respectively (Table 4).

Table 4. Determination of MIC of antifungal compounds on Candida albicans isolated from patients

\section{DISCUSSION}

Candida albicans is the most prevalent fungal pathogen type in humans and the main cause of Candida's vaginitis. It is assumed that approximately $75 \%$ of all women experience vulvovaginal candidiasis (VVC) at least once, with $40-50 \%$ experiencing the infection more than once. Nowadays, candidiasis of vaginitis is treated with nystatin, ketoconazole, and fluconazole $(5,18)$. Although antibiotics do not affect the fungus promotion, they provide a favorable environment for fungal growth through changing the number of fungi and intestinal bacteria (19). The number of antifungal-resistant organisms has risen over the recent years, causing problems in the treatment process. Approximately $10 \%$ of patients with candida vaginitis fail to respond to conventional treatment (6). Given the increased resistance of microorganisms with time, the objectives of this study were to 1) investigate the drug sensitivity of the isolated Candida albicans; 2) report the results to health centers for a better drug administration; 3 ) find a suitable strategy for overcoming this organism; and 4) observe the effect of zinc and synergistic nano- $\mathrm{ZnO}$ combined with fluconazole compared to other antifungal drugs. Falahati et al., Pakshir et al., Nazeri et al., Feglo et al., and Abedzadeh et al. reported that Candida albicans had a higher incidence compared to other Candida species (19-23). De Pádua et al. and Panchal et al. observed that the highest incidence of Candida albicans was $84 \%$ and $55 \%$, respectively $(6,24)$. In the present study, among the 120 subjects suspected of Candida infections, the highest frequency of Candida species belonged to Candida albicans similar to most studies $(41.6 \%)$.

The sensitivity patterns of Candida albicans isolates to fluconazole, nystatin, and amphotericin B were 96\%, 71\%, and 98.8\%, respectively, as reported by Nazeri et al. (22). Pfaller et al. and Nemati Shirazi et al. (2008) found that $90.1 \%$ and $69.8 \%$ of Candida species were sensitive to fluconazole $(25,26)$. Kennedy showed that $98 \%$ of Candida isolates were sensitive to itraconazole (27), which was $96 \%$ in the present study.

Moreover, all Candida strains isolated from patients were $66 \%$ and $100 \%$ sensitive to fluconazole and amphotericin B, respectively. The difference may be attributed to the differences in the studied population or the purity degree of the drugs. 
Furthermore, Candida albicans was $98 \%$ and $60 \%$ sensitive to nicotine and ketoconazole, respectively.

Pasquet et al. introduced zinc as a new preservative with effective antimicrobial activities against bacteria and fungi (28). In 2011, Joushaghani et al. investigated the antifungal effects of zinc and sodium dodecyl sulfate nanoparticles on Candida albicans standard strain compared to fluconazole. The determined MIC range was $296 \pm 013 \mu \mathrm{g} / \mathrm{ml}$ for zinc-oxide nanoparticles, 0.5-0. $001 \mu \mathrm{g} /$ $\mathrm{ml}$ regarding to sodium dodecyl sulfate, and 0.62-0.88 $\mu \mathrm{g} / \mathrm{ml}$ concerning fluconazole. Hosseini et al. showed that the $\mathrm{ZnO}$ nanoparticle had antifungal effects and could be utilized as an appropriate agent to eliminate Candida albicans (29). In present study, the measured MIC range in terms of $\mathrm{ZnO}$ nanoparticles combined with fluconazole was 32-256 $\mu \mathrm{g} \mathrm{/} \mathrm{ml}$. This indicates that the synergistic activity of $\mathrm{ZnO}$ nanoparticle disc with fluconazole had a significant effect on isolated Candida albicans compared with the

\section{REFERENCES}

1. Criseo G, Scordino F, Romeo O. Current methods for identifying clinically important cryptic Candida species. J Microbiol Methods. 2015; 111: 50-6. doi: 10.1016/j.mimet.2015.02.004.

2. Rajabibazl M, Rasaee MJ, Nouri FM, Farahnejad Z. Development of agglutination test for detection of isolated mannoprotein antigen from Candida Albicans. 2013; 11(2): 8995.

3. Ho K-m, Cheng T-s. Common superficial fungal infections-a short review. Med Bull. 2010; 15(11): 23-7.

4. Mohammadzadeh Rostami F, Shalibeik S, Rabi Nezhad Mousavi M. Molecular Characterization and Antibiotic Resistance Pattern of Nosocomial Clinical Isolates in Southeast of Iran. Medical Laboratory Journal. 2020 Jan 10;14(1):20-8.

5. Nasrollahi Z, Yadegari MH. Antifungal activity of caffeine in combination with fluconazole against Candida albicans. Infection, Epidemiology and Microbiology. 2016; 2(2): 18-21.

6. De Pádua RF, Guilhermetti E, Svidzinski TE. In vitro activity of antifungal agents on yeasts isolated from vaginal secretion. Acta Scientiarum. 2003; 25(1): 51-4.

7. Higa LH, Schilrreff P, Perez AP, Morilla MJ, Romero EL. The intervention of nanotechnology against epithelial fungal diseases. Journal of Biomaterials and Tissue Engineering. 2013; 3(1): 70-88.

8. Schmiedel Y, Zimmerli S. Common invasive fungal diseases: an overview of invasive candidiasis, aspergillosis, cryptococcosis, and Pneumocystis pneumonia. Swiss medical weekly. 2016; 146: w14281.

9. Kurtzman C, Fell JW, Boekhout T. The yeasts: a taxonomic study. $5^{\text {th }}$ ed: Elsevier. 2011; 1-292.

10. Hashemi S. Prevalence of candida and non-candida yeasts isolated from patients with yeast fungal infections in Tehran labs. Tehran Univ Med J. 2011; 69 (1) :55-62

11. Evans EGV, Richardson MD. Medical mycology. A practical approach: IRL press; 1989. separate use of $\mathrm{ZnO}$ nanoparticles or fluconazole.

Jehad et al. examined the antimicrobial activity of $\mathrm{ZnO}$ nanoparticles on Candida albicans. They showed that $\mathrm{ZnO}$ nanoparticles had 18-mm inhibition zone (16); meanwhile, in our study, the mean diameters of the inhibition zone of zinc nanoparticles and $\mathrm{ZnO}$ nanoparticles combined with fluconazole were 23.75 and 26.76 , respectively.

\section{CONCLUSION}

Discovering a new antifungal agent against pathogenic yeast species can aid the medical community, particularly gynecologists and midwifery experts, in prescribing medicines and preventing the recurrence and development of resistant strains.

\section{ACKNOWLEDGEMENTS}

This paper has been extracted from project

No.15430507971001 . (Performer:

Mohammad Dakhili).

\section{CONFLICTS OF INTEREST}

None to declare.

12. Hooper RW, Ashcraft DS, Pankey GA. In vitro synergy with fluconazole plus doxycycline or tigecycline against clinical Candida glabrata isolates. Medical mycology. 2018; 57(1): 122-6.

13. Pathak AK, Jain NR, Joshi R. Antibiogram of Candida species isolated from mono and multi-species oral candidal carriage using disk diffusion method. Saudi Journal for Health Sciences. 2012; 1(3): 132-138. DOI: 10.4103/22780521.106082 .

14. Espinel-Ingroff A, Arthington-Skaggs B, Iqbal N, Ellis D, Pfaller M, Messer S, et al. Multicenter evaluation of a new disk agar diffusion method for susceptibility testing of filamentous fungi with voriconazole, posaconazole, itraconazole, amphotericin B, and caspofungin. J Clin Microbiol. 2007; 45(6): 1811-20. DOI: 10.1128/JCM.00134-07.

15. Sharma RK, Ghose R. Synthesis of zinc oxide nanoparticles by homogeneous precipitation method and its application in antifungal activity against Candida albicans. Ceramics International. 2015; 41(1): 967-75.

16. Jehad Yousef M, Enas Danial N. In vitro antibacterial activity and minimum inhibitory concentration of zinc oxide and nano-particle zinc oxide against pathogenic strains. Journal of Health Sciences. 2012; 2(4): 38-42.

17. Stojković D, Soković M, Glamočlija J, Džamić A, Ćirić A, Ristić M, et al. Chemical composition and antimicrobial activity of Vitex agnus-castus L. fruits and leaves essential oils. Food Chemistry. 2011; 128(4): 1017-22.

18. Namazi A, Sahati F, Adibpour M, Mazloomi AS, Mohammad alizadeh S, Babapour J. Prevalence and Predisposing Factors to Candidiasis Infection in Women Supported by Health Centers of Tabriz, 2004. JSSU. 2007; 15(1): 54-60

19. Feglo P, Narkwa P. Prevalence and antifungal susceptibility patterns of yeast isolates at the Komfo Anokye Teaching Hospital (KATH), Kumasi, Ghana. British Microbiology Research Journal. 2012; 2(1):10. 
20. Falahati M, Sharifinia S, Foroumadi A, Bolouri F, Akhlagh L, Yazdan Parast A, et al. Drug resistance pattern in Candida species isolated from vaginitis. Razi Journal of Medical Sciences. 2009; 16(65): 40-45.

21. Pakshir K, Akbarzadeh M, Bonyadpour B, Mohagheghzadeh A. In vitro activity and comparison of clotrimazol, fluconazol and nystatin against Candida vaginitis isolates in Shiraz, 2008. JRUMS. 2010; 9(3): 210-220.

22. Nazeri M, Mesdaghinia E, Moravej SAR, Atabakhshiyan R, Soleymani F. Prevalence of vulvovaginal candidiasis and frequency of candida species in women. Journal of Mazandaran University of Medical Sciences. 2012; 21(86): 254-62.

23. Abedzadeh AH, Dakhili M, Haghighi N, Khalilian M. An Investigation of the Prevalence of Candida Species in Women Referring to Health Center of Qom Province, Iran. Qom University Of Medical Sciences Journal. 2016; 9(12): 65-71.

24. Panchal P, Katara R, Mehta R, Soni S, Nanera A, Trivedi N, et al. Microbiological study of various candida species and its antifungal sensitivity testing isolated from antenatal women with vaginitis, in tertiary care teaching hospital, western india. International Journal of Microbiology Research. 2013; 5(6): 486.

25. Pfaller MA, Messer SA, Hollis RJ, Boyken L, Tendolkar S, Kroeger $\mathrm{J}$, et al. Variation in susceptibility of bloodstream isolates of Candida glabrata to fluconazole according to patient age and geographic location in the United States in 2001 to 2007. J Clin Microbiol. 2009; 47(10): 3185-90. doi: 10.1128/JCM.00946-09.
26. Shirzi NL, Shams Ghahfarokhi M, Yadgari MH. Evaluation of disk diffusion and broth microdilution methods for fluconazole susceptibility testing in one group of Candida spp. in Tehran. Daneshvar Medicine. 2008; 15(73): 51-58.

27. Kennedy H, Shankland G, Bagg J, Chalmers E, Gibson B, Williams C. Fluconazole and itraconazole susceptibilities of Candida spp. isolated from oropharyngeal specimens and blood cultures of paediatric haematology/oncology patients. Mycoses. 2006; 49(6): 457-62 DOI: 10.1111/j.1439-0507.2006.01272.x.

28. Pasquet J, Chevalier Y, Couval E, Bouvier D, Bolzinger MA. Zinc oxide as a new antimicrobial preservative of topical products: Interactions with common formulation ingredients. Int J Pharm. 2015; 479(1): 88-95. doi: 10.1016/j.ijpharm.2014.12.031

29. Hosseini SS, Mohammadi SR, Joshaghani HR, Eskandari M. Antifungal effect of sodium dodecil sulfate and nano particle $\mathrm{ZnO}$ on growth inhibition of standard strain of Candida albicans. J Gorgan Univ Med Sci. 2011; 12(4): 64-69. 